\title{
Effect of osteopontin on the mRNA expression of ADAMTS4 and ADAMTS5 in chondrocytes from patients with knee osteoarthritis
}

\author{
SHU-GUANG GAO ${ }^{1,2^{*}}$, CHAO ZENG $^{1 *}$, YANG SONG $^{1,3^{*}}$, JIAN TIAN $^{1}$, CHAO CHENG $^{1}$, \\ TUO YANG ${ }^{1}$, HUI LI ${ }^{1}$, FANG-JIE ZHANG ${ }^{1}$ and GUANG-HUA LEI ${ }^{1,2}$ \\ ${ }^{1}$ Department of Orthopedics, Xiangya Hospital; ${ }^{2}$ Orthopedics Institute, Central South University, Changsha, \\ Hunan 410008; ${ }^{3}$ Department of Orthopedics, Linyi People's Hospital, Linyi, Shandong 276001, P.R. China
}

Received July 16, 2014; Accepted February 11, 2015

DOI: $10.3892 / \mathrm{etm} .2015 .2310$

\begin{abstract}
Previous studies have demonstrated that osteopontin (OPN) levels are elevated in the synovial fluid and articular cartilage, and are associated with the severity of knee osteoarthritis (OA). However, the role of OPN in the pathogenesis of OA has yet to be elucidated. The present study aimed to investigate the effects of OPN on the expression of the aggrecanases, a disintegrin and metalloproteinase with thrombospondin motifs (ADAMTS) 4 and ADAMTS5, in human OA chondrocytes, as they serve a key function in aggrecan degradation. Human OA chondrocytes were obtained from the knees of 16 patients with OA, and subsequently cultured in a monolayer. The chondrocytes were divided into three groups, which included the control (no treatment), N-OPN (treated with $100 \mathrm{ng} / \mathrm{ml}$ OPN, the normal circulating OPN concentration) and the H-OPN groups (treated with $1 \mu \mathrm{g} / \mathrm{ml} \mathrm{OPN}$, a high OPN concentration). Reverse transcription-quantitative polymerase chain reaction was performed to quantify the relative mRNA expression levels of ADAMTS4, ADAMTS5 and aggrecan in the chondrocytes. The mRNA expression levels of ADAMTS4 were significantly reduced in the N-OPN and $\mathrm{H}$-OPN groups when compared with the control group $(\mathrm{P}<0.0001)$. In addition, the mRNA expression levels of ADAMTS4 were lower in the H-OPN group when compared with the N-OPN group $(\mathrm{P}<0.001)$. However, no statistically significant difference was observed in the relative mRNA expression levels of ADAMTS5 among the three groups $(P>0.05)$. Furthermore, the mRNA expression levels of aggrecan were higher in the $\mathrm{N}-\mathrm{OPN}$ and $\mathrm{H}-\mathrm{OPN}$ groups when
\end{abstract}

Correspondence to: Professor Guang-Hua Lei, Department of Orthopedics, Xiangya Hospital, Central South University, 87 Xiangya Road, Changsha, Hunan 410008, P.R. China

E-mail:1gh9640@gmail.com

*Contributed equally

Key words: osteopontin, osteoarthritis, a disintegrin and metalloproteinase with thrombospondin motifs 4 , a disintegrin and metalloproteinase with thrombospondin motifs 5 , aggrecan compared with the control group $(\mathrm{P}<0.0001)$, and a statistically significant difference was observed between the N-OPN and $\mathrm{H}-\mathrm{OPN}$ groups with regard to the mRNA expression of aggrecan $(\mathrm{P}<0.0001)$. These results demonstrated that OPN may exert a protective effect in human OA chondrocytes against aggrecan degradation by suppressing the expression of ADAMTS4.

\section{Introduction}

Osteoarthritis $(\mathrm{OA})$ is the most common degenerative disorder of the joint, which is characterized by the progressive loss of articular cartilage. This loss is attributed primarily to the proteolysis of certain structural components within the extracellular matrix (ECM), including proteoglycans and collagens (1-3). Aggrecan is a major component of the cartilage-specific proteoglycans. A number of studies have indicated that the aggrecanase-mediated degradation of aggrecan is a crucial factor in the development of OA (4-7). Aggrecanases are members of the A disintegrin and metalloproteinase with thrombospondin motifs (ADAMTS) family, which includes ADAMTS1, 4, 5, 8,9 and 15, that are expressed in the articular cartilage tissues and chondrocytes of numerous animal species, in addition to humans (8). A previous study indicated that ADAMTS4 and ADAMTS5 were responsible for aggrecan degradation in a human model of OA (9).

Osteopontin (OPN) is an ECM glycoprotein that is hypothesized to be a potential inflammatory cytokine. OPN, as a biomarker, has been studied in vivo in rats (10), in healthy humans and patients with OA $(11,12)$ and in patients with rheumatoid arthritis (13). Previous studies observed that the mRNA expression levels of OPN were enhanced in the cartilage of individuals with OA when compared with the normal cartilage $(11,12)$. Thus, OPN may be involved in the molecular pathogenesis of OA, contributing to the progressive degeneration of articular cartilage (10-12). Kon et al (14) revealed that the function of OPN in inflammatory processes was regulated by syndecan-4, a key sulfated glycosaminoglycan that is located on the cell surface. Furthermore, Matsui et al (10) indicated that a deficiency in OPN resulted in the induction of matrix metalloproteinase (MMP)-13, which degrades type II collagen, a major structural component of the cartilage matrix. 
However, the effects of OPN on aggrecanases in human OA chondrocytes are yet to be fully elucidated. Therefore, the present study aimed to determine the effects of OPN on the expression levels of ADAMTS4 and ADAMTS5 in human OA chondrocytes.

\section{Materials and methods}

Study sample. Fragments of articular cartilage were obtained from the femoral condyles of 16 patients with knee OA, who had undergone total knee replacement surgery. The diagnosis of knee OA was determined according to the clinical and radiological criteria of the American College of Rheumatology (15). The mean age of the patients was $64.1 \pm 5.8$ years, with an age range of 48-73 years. The experimental protocols were approved by the Institutional Review Board at Xiangya Hospital of Central South University (Changsha, China) and informed consent was obtained from each patient. A histological examination of the representative cartilage samples identified typical OA characteristics, including the presence of chondrocyte clusters, loss of metachromasia and fibrillation.

Chondrocyte cultures. Cartilage samples were dissected aseptically and cut into small pieces following surgery. The fragments were washed in Hyclone Dulbecco's modified Eagle's medium (DMEM; GE Healthcare Life Sciences, Logan, UT, USA) without phenol red, that was supplemented with $2 \%$ penicillin/streptomycin solution and $0.2 \%$ Amphotericin B (Gibco Life Technologies, Grand Island, NY, USA). The chondrocytes were isolated from the articular cartilage by sequential enzymatic digestion. Briefly, the samples were treated with $0.1 \%$ hyaluronidase for $30 \mathrm{~min}, 0.5 \%$ pronase for $1 \mathrm{~h}$ and $0.2 \%$ collagenase for $1 \mathrm{~h}$ (Gibco Life Technologies) at $37^{\circ} \mathrm{C}$ in a wash solution containing DMEM, penicillin/streptomycin solution and Amphotericin B. Subsequently, the cell suspension was filtered twice through a $70-\mu$ m nylon mesh (USCN Life Science Inc., Wuhan, China), washed and centrifuged at $150 \mathrm{x} \mathrm{g}$ for $10 \mathrm{~min}$ at $4^{\circ} \mathrm{C}$. A Trypan Blue (USCN Life Science Inc.) viability test indicated that $94.47 \pm 1.93 \%$ of the cells were recovered alive. Primary chondrocyte cultures were maintained in an atmosphere of $5 \% \mathrm{CO}_{2}$ at $37^{\circ} \mathrm{C}$ for two weeks.

Treatments. Chondrocytes in the first passage were seeded in 24-well plates at a starting density of $1 \times 10^{4}$ cells/well, until confluency was achieved. Next, the cell population was divided into three groups: i) The control group, maintained as unstimulated and untreated control cells; ii) the normal concentration (N-OPN) group that were treated with $100 \mathrm{ng} / \mathrm{ml}$ OPN (R\&D Systems, Inc., Minneapolis, MN, USA) for $48 \mathrm{~h}$, which was within the normal circulating concentration range (2.3-200 ng/ml) described in previous studies (12,16-19); and iii) the high concentration (H-OPN) group, treated with $1 \mu \mathrm{g} / \mathrm{ml}$ OPN for $48 \mathrm{~h}$.

Cell viability assay. Following treatment with OPN for $48 \mathrm{~h}$, the cell viability was determined using a colorimetric MTT assay. At one day prior to OPN treatment, the cells were seeded into 96-well plates. After $48 \mathrm{~h}$ of OPN treatment, the culture medium was removed and $20 \mu \mathrm{l}$ MTT solution $(5 \mathrm{mg} / \mathrm{ml}$, in phosphate-buffered saline; USCN Life Science Inc.) was added to each well and incubated in conditions of $5 \% \mathrm{CO}_{2}$ at $37^{\circ} \mathrm{C}$ for $4 \mathrm{~h}$. The supernatant was subsequently aspirated, and the formazan reaction products were dissolved in dimethyl sulfoxide (Sigma-Aldrich, St. Louis, MO, USA) solution and agitated for $15 \mathrm{~min}$. Spectrophotometric absorbance values were measured at $570 \mathrm{~nm}$ using a Multiskan MK3 ELISA plate reader (Thermo Fisher Scientific, Inc., Waltham, MA, USA).

Total RNA isolation, quantification and reverse transcription. Following the various treatments, the chondrocytes were lysed and the total RNA was extracted using TRIzol reagent (Invitrogen Life Technologies, Rockville, MD, USA), according to the manufacturer's instructions. The total RNA was quantified using a NanoDrop 1000 spectrophotometrer (Thermo Fisher Scientific, Wilmington, DE, USA). A $1-\mu \mathrm{g}$ sample of total RNA was converted to cDNA using the All-in-One ${ }^{\mathrm{TM}}$ First Strand cDNA Synthesis kit (AORT-0020; GeneCopoeia, Inc., Rockville, MD, USA). For each reaction, $4 \mu 1$ 5X RT reaction buffer, $1 \mu 1$ RNase inhibitor $(20 \mathrm{U} / \mu \mathrm{l})$, $2 \mu 1 \mathrm{dNTP}$ Mix (10 mM), $1 \mu \mathrm{l}$ M-MLV Reverse Transcriptase (200 U/ $\mu \mathrm{l})$ and sterile water were added to the RNA, with a final volume of $20 \mu \mathrm{l}$. The reaction system was incubated for $60 \mathrm{~min}$ at $42^{\circ} \mathrm{C}$ and then at $70^{\circ} \mathrm{C}$ for $5 \mathrm{~min}$. The final cDNA products were stored in aliquots at $-20^{\circ} \mathrm{C}$ until required.

Quantitative polymerase chain reaction ( $q P C R)$. Primers were synthesized by Shanghai Sangon Biological Engineering Technology \& Services Co., Ltd. (Shanghai, China), and the sequences were as follows: ADAMTS4 forward, 5'-ATGGCT ATGGGCACTGTCTC-3' and reverse, 5'-GTGTTTGGTCTG GCACATGG-3'; ADAMTS5 forward, 5'-CCTGCCCACCCA ATGGTAAATC-3' and reverse, 5'-CGGCCTACATTCAGT GCCATC-3'; aggrecan forward, 5'-CCTGGTGTGGCTGCT GTC-3' and reverse, 5'-CTGGCTCGGTGGTGAACTC-3'; $\beta$-actin forward, 5'-GGAAATCGTGCGTGACATTA-3' and reverse, 5'-GGAGCAATGATCTTGATCTTC-3'.

The All-in-One ${ }^{\mathrm{TM}} \mathrm{qPCR}$ Mix was used for universal qPCR (AOPR-0200; GeneCopoeia, Inc.), and the reaction system contained 12.5 $\mu \mathrm{l} 2 \mathrm{X}$ qPCR Master Mix, $2.5 \mu \mathrm{l}$ forward primer $(0.3 \mu \mathrm{M}), 2.5 \mu \mathrm{l}$ reverse primer $(0.3 \mu \mathrm{M}), 2 \mu \mathrm{l}$ cDNA template and $5.5 \mu \mathrm{l}$ sterile water, with a final volume of $25 \mu \mathrm{l}$. SYBR Green qPCR SuperMix (GeneCopoeia, Inc.) and an ABI 7900 Sequence Detection System (Applied Biosystems Life Technologies, Foster City, CA, USA) were used for all the qPCR assays. The qPCR thermal cycling conditions were as follows: Initial denaturation at $95^{\circ} \mathrm{C}$ for $10 \mathrm{~min}$, followed by 40 cycles of denaturation at $95^{\circ} \mathrm{C}$ for $15 \mathrm{sec}$, annealing at $60^{\circ} \mathrm{C}$ for $30 \mathrm{sec}$ and extension at $72^{\circ} \mathrm{C}$ for $30 \mathrm{sec}$. Melting curve analysis was performed following the final amplification period via a temperature gradient of $95^{\circ} \mathrm{C}$ for $15 \mathrm{sec}, 60^{\circ} \mathrm{C}$ for $15 \mathrm{sec}$ and $95^{\circ} \mathrm{C}$ for $15 \mathrm{sec}$.

The mRNA expression levels of ADAMTS4, ADAMTS5 and aggrecan were normalized against the levels of $\beta$-actin. Relative quantification analysis was performed using the $2^{-\Delta \Delta \mathrm{Ct}}$ method.

Statistical analysis. Results are expressed as the mean \pm standard deviation. Statistical analysis was performed using SPSS software, version 17.0 (SPSS, Inc., Chicago, IL, USA). 
Analysis of variance and Fisher's least significant difference t-test were used for statistical evaluation among the groups, where $\mathrm{P}<0.05$ was considered to indicate a statistically significant difference.

\section{Results}

Effect of OPN on chondrocyte viability in vitro. Table I shows the MTT data as fraction ratios relative to the control values. No statistically significant difference was observed in the cell viability of the three groups after $48 \mathrm{~h}$ of incubation. These results indicated that OPN did not suppress the survival of human chondrocytes in vitro.

Effect of OPN on the mRNA expression levels of ADAMTS4. As shown in Table II, the relative mRNA expression levels of ADAMTS4 were significantly lower in the N-OPN group

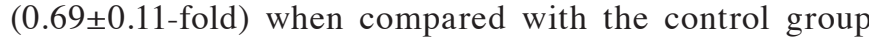
$(\mathrm{P}<0.0001)$. In addition, the mRNA expression levels of ADAMTS4 were significantly decreased in the H-OPN group $(0.58 \pm 0.12$-fold $)$ when compared with the control group $(\mathrm{P}<0.0001)$. A statistically significant difference was observed between the N-OPN and $\mathrm{H}-\mathrm{OPN}$ groups with regard to the mRNA expression levels of ADAMTS4 $(\mathrm{P}=0.009)$.

Effect of OPN on the mRNA expression of ADAMTS5. No statistically significant differences were observed in the relative mRNA expression levels of ADAMTS5 when comparing the N-OPN and H-OPN groups $(1.12 \pm 0.53$ and $1.38 \pm 0.97$-fold, respectively) with the control group. Furthermore, a statistically significant difference was not identified between the $\mathrm{N}-\mathrm{OPN}$ and $\mathrm{H}-\mathrm{OPN}$ groups $(\mathrm{P}>0.05$; Table III).

Effect of OPN on the mRNA expression levels of aggrecan. Relative mRNA expression levels of aggrecan were increased in the N-OPN $(2.82 \pm 1.18$-fold) and H-OPN (3.73 \pm 0.61 -fold) groups when compared with the control group $(\mathrm{P}<0.0001)$. In addition, the relative aggrecan mRNA expression levels in the $\mathrm{H}-\mathrm{OPN}$ group were higher compared with the N-OPN group $(\mathrm{P}<0.0001 ;$ Table IV).

\section{Discussion}

To the best of our knowledge, the present study is the first to demonstrate that OPN suppresses aggrecan degradation by downregulating ADAMTS4 expression in human OA chondrocytes. To date, there have been contradictory findings with regard to the expression of aggrecan-degrading members of the ADAMTS family. Previous studies investigating arthritis and OA with ADAMTS4/5 knockout mice have revealed that inhibition of ADAMTS5, but not ADAMTS4, mitigates aggrecan degradation and cartilage destruction, suggesting that ADAMTS5 has an important function in aggrecan degradation in mice (20-22). However, in human chondrocytes, ADAMTS4 is upregulated by interleukin (IL)-1, tumor necrosis factor $\alpha$, oncostatin $\mathrm{M}$ and transforming growth factor $\beta$, indicating that ADAMTS4 functions as the primary aggrecanase in human OA cartilage $(4,8,9,23-25)$. The results of the present study, with regard to the mRNA expression of ADAMTS4 in human OA chondrocytes under monolayer culture, provide the first indi-
Table I. Cell viability of each group.

\begin{tabular}{lc}
\hline Group & Chondrocyte viability in vitro $(\%)$ \\
\hline Control & $98.22 \pm 1.06$ \\
N-OPN $(100 \mathrm{ng} / \mathrm{ml})$ & $97.05 \pm 1.11$ \\
H-OPN $(1 \mu \mathrm{g} / \mathrm{ml})$ & $97.81 \pm 0.91$
\end{tabular}

$\mathrm{P}>0.05$, comparison among the groups. OPN, osteopontin; N-OPN, normal OPN concentration; H-OPN, high OPN concentration.

Table II. Relative mRNA expression levels of ADAMTS4.

\begin{tabular}{lc}
\hline Group & Relative fold mRNA level \\
\hline Control & 1 \\
N-OPN $(100 \mathrm{ng} / \mathrm{ml})$ & $0.69 \pm 0.11^{\mathrm{a}}$ \\
H-OPN $(1 \mu \mathrm{g} / \mathrm{ml})$ & $0.58 \pm 0.12^{\mathrm{ab}}$ \\
\hline
\end{tabular}

${ }^{\mathrm{a}} \mathrm{P}<0.0001$, vs. control and ${ }^{\mathrm{b}} \mathrm{P}=0.009$, vs. N-OPN. ADAMTS4, a disintegrin and metalloproteinase with thrombospondin motifs 4; OPN, osteopontin; N-OPN, normal OPN concentration; H-OPN, high OPN concentration.

Table III. Relative mRNA expression levels of ADAMTS5.

\begin{tabular}{lc}
\hline Group & Relative fold mRNA level \\
\hline Control & 1 \\
N-OPN $(100 \mathrm{ng} / \mathrm{ml})$ & $1.12 \pm 0.53$ \\
H-OPN $(1 \mu \mathrm{g} / \mathrm{ml})$ & $1.38 \pm 0.97$ \\
\hline
\end{tabular}

$\mathrm{P}>0.05$, comparison among the groups. ADAMTS5, a disintegrin and metalloproteinase with thrombospondin motifs 5; OPN, osteopontin; N-OPN, normal OPN concentration; H-OPN, high OPN concentration.

Table IV. Relative mRNA expression levels of aggrecan.

\begin{tabular}{lc}
\hline Group & Relative fold mRNA level \\
\hline Control & 1 \\
N-OPN $(100 \mathrm{ng} / \mathrm{ml})$ & $2.82 \pm 1.18^{\mathrm{a}}$ \\
H-OPN $(1 \mu \mathrm{g} / \mathrm{ml})$ & $3.73 \pm 0.61^{\mathrm{ab}}$ \\
\hline
\end{tabular}

${ }^{\mathrm{a}} \mathrm{P}<0.0001$, vs. control and ${ }^{\mathrm{b}} \mathrm{P}<0.0001$, vs. N-OPN. OPN, osteopontin; N-OPN, normal OPN concentration; H-OPN, high OPN concentration.

cations that OPN selectively inhibits ADAMTS4 expression, while the expression of ADAMTS5 is not affected by OPN.

Previous studies have confirmed the immunohistochemical expression of OPN in synovial lining cells (26), fibroblasts in the synovial tissues (20) and articular chondrocytes (27). Cell adhesion, migration or inflammation have 
been hypothesized to be involved in the release of OPN (28). Attur et al (29) observed that the mRNA expression of OPN was highly upregulated in OA cartilage, as compared with normal cartilage. In addition, the authors indicated that the addition of recombinant OPN to human OA-affected cartilage under ex vivo conditions suppressed the spontaneous production of nitric oxide and prostaglandin E2. These observations demonstrated that overexpression of OPN is present in OA cartilage, and functions as an endogenous inhibitor of the production of inflammatory mediators in the cartilage. Furthermore, $\mathrm{Xu}$ et al (30) reported that overexpression of OPN induced the expression of proinflammatory chemokines and cytokines, including IL-1, IL-8, CXCL1 and CCL2, and activated the nuclear factor $(\mathrm{NF})-\kappa \mathrm{B}$ signaling pathway. Therefore, increased expression of OPN may be associated with the pathogenesis of OA.

Matsui et al (10) demonstrated that structural changes and an increase in the loss of proteoglycan from the cartilage tissue were elevated when OPN was absent. In addition, an OPN deficiency was shown to result in the induction of MMP-13, which degrades type II collagen, a major component of the cartilage matrix. Matsui et al additionally demonstrated that OPN functioned as a critical intrinsic regulator of cartilage degradation and that OPN deficiency resulted in the accelerated development of OA-like tenascin-C deficiency. In the study by Okamura et al (31), the rate of cartilage repair in tenascin-C knockout mice was shown to be significantly reduced when compared with wild type mice. In addition, tenascin- $\mathrm{C}$ deficiency was demonstrated to progress with cartilage degeneration. Nakoshi et al (32) observed that the distribution of tenascin- $\mathrm{C}$ was associated with chondroitin sulfate production and chondrocyte proliferation in OA cartilage. The study also found that tenascin-C affected DNA synthesis, proteoglycan content and the mRNA expression of aggrecan in vitro. The authors hypothesized that tenascin- $\mathrm{C}$ may be involved in the repair of human OA cartilage. Collectively, these observations and those of previous studies $(12,33)$ indicate that the remodeling of cartilage may be involved in the elevation of OPN levels.

Cook et al (34) revealed that OPN was able to regulate hyaluronan (HA) production in breast cancer cells. Furthermore, Tajima et al (35) indicated that OPN was involved in multidrug resistance by enhancing the binding of CD44 to HA. Ariyoshi et al (36) demonstrated disruptive changes in chondrocyte-matrix interactions by HA oligosaccharides, which induced matrix degradation and elevated levels of aggrecanase (ADAMTS4 and ADAMTS5) activity via the $\mathrm{NF}-\kappa \mathrm{B}$ signaling pathway. Yatabe et al (4) observed that HA2700 (2,700 kDa HA) suppressed aggrecan degradation in OA chondrocytes via the downregulation of IL-1 $\alpha$-induced ADAMTS4 expression through the CD44 and intracellular adhesion molecule 1 signaling pathways. These results suggested that OPN may suppress aggrecanase degradation through the direct inhibition of ADAMTS4, by enhancing the binding of CD44 to HA. This OPN-mediated mechanism may serve a key function in the progression of OA. Currently, further investigations are being undertaken with the aim of defining the signaling events induced by OPN and determining potential experimental strategies to inhibit the development of OA.
In conclusion, the results of the present study indicate that OPN is involved in the progression of OA, and that the activation of OPN in chondrocytes may prevent aggrecan degradation by suppressing ADAMTS4 expression. As a result of OPN activation, a reduction was observed in the rate of cartilage degradation. Thus, OPN appears to function as a protective factor during the progression of OA. Additional experiments are currently attempting to further define the association between OPN and proinflammatory signaling pathways in human OA chondrocytes. The results of the present study can be used to evaluate whether transplantation of OPN-overexpressing chondrocytes into OA cartilage may provide a useful local and transient therapeutic approach for the prevention of joint inflammation and the support of cartilage repair. Therefore, OPN may offer a novel therapeutic approach for the treatment of OA.

\section{Acknowledgements}

This study was supported by the National Natural Science Foundation of China (no. 81201420, 81272034 and 81472130), the Provincial Science Foundation of Hunan (no. 14JJ3032), the Scientific Research Project of the Development and Reform Commission of Hunan Province ([2013]1199), the Scientific Research Project of Science and Technology Office of Hunan Province (no. 2013SK2018) and the Doctoral Scientific Fund Project of the Ministry of Education of China (no. 20120162110036).

\section{References}

1. Bijlsma JW, Berenbaum F and Lafeber FP: Osteoarthritis: an update with relevance for clinical practice. Lancet 377: 2115-2126, 2011.

2. Jiang L, Rong J, Zhang Q, et al: Prevalence and associated factors of knee osteoarthritis in a community-based population in Heilongjiang, Northeast China. Rheumatol Int 32: 1189-1195, 2012.

3. Bobacz K, Erlacher L, Smolen J, Soleiman A and Graninger WB: Chondrocyte number and proteoglycan synthesis in the aging and osteoarthritic human articular cartilage. Ann Rheum Dis 63: 1618-1622, 2004.

4. Yatabe T, Mochizuki S, Takizawa M, et al: Hyaluronan inhibits expression of ADAMTS4 (aggrecanase-1) in human osteoarthritic chondrocytes. Ann Rheum Dis 68: 1051-1058, 2009.

5. Cheung KS, Hashimoto K, Yamada N and Roach HI: Expression of ADAMTS-4 by chondrocytes in the surface zone of human osteoarthritic cartilage is regulated by epigenetic DNA de-methylation. Rheumatol Int 29: 525-534, 2009.

6. Larsson S, Englund M, Struglics A and Lohmander LS: Association between synovial fluid levels of aggrecan ARGS fragments and radiographic progression in knee osteoarthritis. Arthritis Res Ther 12: R230, 2010.

7. Huang K and Wu LD: Suppression of aggrecanase: a novel protective mechanism of dehydroepiandrosterone in osteoarthritis? Mol Biol Rep 37: 1241-1245, 2010.

8. Naito S, Shiomi T, Okada A, et al: Expression of ADAMTS4 (aggrecanase-1) in human osteoarthritic cartilage. Pathol Int 57: 703-711, 2007.

9. Verma P and Dalal K: ADAMTS-4 and ADAMTS-5: key enzymes in osteoarthritis. J Cell Biochem 112: 3507-3514, 2011.

10. Matsui Y, Iwasaki N, Kon S, et al: Accelerated development of aging-associated and instability-induced osteoarthritis in osteopontin-deficient mice. Arthritis Rheum 60: 2362-2371, 2009.

11. Pullig O, Weseloh G, Gauer S and Swoboda B: Osteopontin is expressed by adult human osteoarthritic chondrocytes: protein and mRNA analysis of normal and osteoarthritic cartilage. Matrix Biol 19: 245-255, 2000. 
12. Gao SG, Li KH, Zeng KB, Tu M, Xu M and Lei GH: Elevated osteopontin level of synovial fluid and articular cartilage is associated with disease severity in knee osteoarthritis patients. Osteoarthritis Cartilage 18: 82-87, 2010.

13. Sharif SA, Du X, Myles T, et al: Thrombin-activatable carboxypeptidase B cleavage of osteopontin regulates neutrophil survival and synoviocyte binding in rheumatoid arthritis. Arthritis Rheum 60: 2902-2912, 2009.

14. Kon S, Ikesue M, Kimura C, et al: Syndecan-4 protects against osteopontin-mediated acute hepatic injury by masking functional domains of osteopontin. J Exp Med 205: 25-33, 2008

15. Altman R, Asch E, Bloch D, et al: Development of criteria for the classification and reporting of osteoarthritis. Classification of osteoarthritis of the knee. Diagnostic and Therapeutic Criteria Committee of the American Rheumatism Association. Arthritis Rheum 29: 1039-1049, 1986.

16. Chen YJ, Shen JL, Wu CY, Chang YT, Chen CM and Lee FY: Elevated plasma osteopontin level is associated with occurrence of psoriasis and is an unfavorable cardiovascular risk factor in patients with psoriasis. J Am Acad Dermatol 60: 225-230, 2009

17. Denhardt DT, Giachelli CM and Rittling SR: Role of osteopontin in cellular signaling and toxicant injury. Annu Rev Pharmacol Toxicol 41: 723-749, 2001.

18. Ramaiah SK and Rittling S: Role of osteopontin in regulating hepatic inflammatory responses and toxic liver injury. Expert Opin Drug Metab Toxicol 3: 519-526, 2007.

19. Ermakov S, Leonov A, Trofimov S, Malkin I and Livshits G: Quantitative genetic study of the circulating osteopontin in community-selected families. Osteoporos Int 22: 2261-2271, 2011

20. Glasson SS, Askew R, Sheppard B, et al: Deletion of active ADAMTS5 prevents cartilage degradation in a murine model of osteoarthritis. Nature 434: 644-648, 2005.

21. Stanton H, Rogerson FM, East CJ, et al: ADAMTS5 is the major aggrecanase in mouse cartilage in vivo and in vitro. Nature 434 648-652, 2005

22. Koshy PJ, Lundy CJ, Rowan AD, et al: The modulation of matrix metalloproteinase and ADAM gene expression in human chondrocytes by interleukin-1 and oncostatin M: a time-course study using real-time quantitative reverse transcription-polymerase chain reaction. Arthritis Rheum 46: 961-967, 2002.

23. Moulharat N, Lesur C, Thomas M, et al: Effects of transforming growth factor-beta on aggrecanase production and proteoglycan degradation by human chondrocytes in vitro. Osteoarthritis Cartilage 12: 296-305, 2004.

24. Hui W, Barksby HE, Young DA, et al: Oncostatin M in combination with tumour necrosis factor alpha induces a chondrocyte membrane-associated aggrecanase that is distinct from ADAMTS aggrecanase-1 or -2. Ann Rheum Dis 64: 1624-1632, 2005.
25. Tortorella MD, Malfait AM, Deccico C and Arner E: The role of ADAM-TS4 (aggrecanase-1) and ADAM-TS5 (aggrecanase-2) in a model of cartilage degradation. Osteoarthritis Cartilage 9: 539-552, 2001

26. Ohshima S, Yamaguchi N, Nishioka K, et al: Enhanced local production of osteopontin in rheumatoid joints. J Rheumatol 29: 2061-2067, 2002.

27. Petrow PK, Hummel KM, Schedel J, et al: Expression of osteopontin messenger RNA and protein in rheumatoid arthritis: effects of osteopontin on the release of collagenase 1 from articular chondrocytes and synovial fibroblasts. Arthritis Rheum 43: 1597-1605, 2000.

28. Standal T, Borset M and Sundan A: Role of osteopontin in adhesion, migration, cell survival and bone remodeling. Exp Oncol 26: 179-184, 2004.

29. Attur MG, Dave MN, Stuchin S, Kowalski AJ, Steiner G, Abramson SB, Denhardt DT and Amin AR: Osteopontin: An intrinsic inhibitor of inflammation in cartilage. Arthritis Rheum 44: 578-584, 2001

30. Xu G, Nie H, Li N, et al: Role of osteopontin in amplification and perpetuation of rheumatoid synovitis. J Clin Invest 115: 1060-1067, 2005.

31. Okamura N, Hasegawa M, Nakoshi Y, et al: Deficiency of tenascin-C delays articular cartilage repair in mice. Osteoarthritis Cartilage 18: 839-848, 2010.

32. Nakoshi Y, Hasegawa M, Akeda K, Iino T, Sudo A, Yoshida T and Uchida A: Distribution and role of tenascin-C in human osteoarthritic cartilage. J Orthop Sci 15: 666-673, 2010

33. Gao SG, Cheng L, Zeng C, et al: Usefulness of specific OA biomarkers, thrombin-cleaved osteopontin, in the posterior cruciate ligament OA rabbit model. Osteoarthritis Cartilage 21: 144-150, 2013.

34. Cook AC, Chambers AF, Turley EA and Tuck AB: Osteopontin induction of hyaluronan synthase 2 expression promotes breast cancer malignancy. J Biol Chem 281: 24381-24389, 2006.

35. Tajima K, Ohashi R, Sekido Y, Hida T, Nara T, Hashimoto M, Iwakami S, Minakata K, Yae T, Takahashi F et al: Osteopontin-mediated enhanced hyaluronan binding induces multidrug resistance in mesothelioma cells. Oncogene 29: 1941-1951, 2010

36. Ariyoshi W, Takahashi N, Hida D, Knudson CB and Knudson W: Mechanisms involved in enhancement of the expression and function of aggrecanases by hyaluronan oligosaccharides. Arthritis Rheum 64: 187-197, 2012. 